\title{
Perturbation of 11-eicosenoate metabolism in female diabetic rats
}

\author{
WILLY J. MALAISSE ${ }^{1}$, LAURENCE PORTOIS ${ }^{2}$, ABDULLAH SENER $^{1}$ and YVON A. CARPENTIER ${ }^{2}$ \\ ${ }^{1}$ Laboratory of Experimental Hormonology and ${ }^{2}$ L. Deloyers Laboratory for \\ Experimental Surgery, Brussels Free University, Brussels, Belgium
}

Received April 8, 2008; Accepted May 8, 2008

\begin{abstract}
Considering the proposed preventive effect of nervonic acid on obesity- and diabetes-related coronary risk factors, the content of its precursors (oleic, 11-eicosenoic and 13-docosenoic acids) was measured in liver and plasma phospholipids and triglycerides, brain and spleen phospholipids, and adipose tissue lipids of fed or overnight fasted control and hereditarily diabetic Goto-Kakizaki female rats, as well as fed streptozotocin-induced diabetic female rats. In liver and brain phospholipids, the 11-eicosenoate/oleate ratio was significantly higher in diabetic rats than in control animals. Such was not the case in either spleen phospholipids or liver triglycerides and adipose tissue lipids. The increase in the liver phospholipid 11-eicosenoate/oleate ratio found in female diabetic rats represents a mirror image of the situation recently documented, in the same animal models of diabetes, in male rats. These contrasting findings may be relevant to the higher coronary heart disease risk prevailing in female, as compared to male, diabetic subjects.
\end{abstract}

\section{Introduction}

Nervonic acid (C24:1w9) is currently considered to participate in the beneficial effects of long-chain polyunsaturated $\omega 3$ fatty acids on diabetes- and obesity-related risk factors (1). In the light of such a proposal, the present study aimed at investigating the content of liver, brain, spleen and plasma phospholipids and triglycerides, as well as adipose tissue

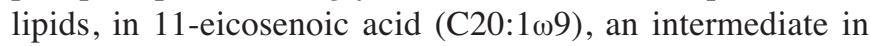
the stepwise conversion of oleic acid $(\mathrm{C} 18: 1 \omega 9)$ to nervonic acid. The paired C20:1 $\omega 9 / \mathrm{C} 18: 1 \omega 9$ ratio was also established in each case. These measurements were conducted in fed or overnight fasted female control rats, female Goto-Kakizaki (GK) rats and male GK rats, as well as fed female streptozotocin-induced diabetic rats (STZ rats). The present study

Correspondence to: Professor W.J. Malaisse, Laboratory of Experimental Hormonology, Brussels Free University, 808 Route de Lennik, B-1070 Brussels, Belgium

E-mail: malaisse@ulb.ac.be

Key words: Goto-Kakizaki rats, streptozotocin-induced diabetic rats, 11-eicosenoate, liver, brain, spleen and plasma phospholipids, triglycerides thus concerns the effect of the nutritional status (fed or fasted rats) in control and GK rats, the gender difference in GK rats, and the situation found in animal models of either Type 1 (STZ rats) or Type 2 (GK rats) diabetes.

\section{Materials and methods}

The identity of the 22 rats examined in the present study, as well as information on such variables as body weight and plasma D-glucose and insulin concentrations, were previously reported (2). Likewise, the fatty acid content and pattern of phospholipids and triglycerides in liver, brain, spleen, adipose tissue and plasma of these 22 rats were already described in prior publications (2-6). The two variables investigated in the present study were not considered, however, in these prior reports.

The methods for extraction and characterization of lipids were also described in previous publications (7).

All results are here presented as mean values $( \pm$ SEM, or range of individual variations whenever $n=2$ ), together with the number of individual values (n) or degree of freedom (d.f.). The statistical significance of differences between mean values was assessed by the Student's t-test or, when required, by covariance analysis. Only those samples containing a sizeable amount of C20:1 $\omega 9$ are taken into consideration, except for the results illustrated in Fig. 2 which refer to all available measurements including null values.

\section{Results}

Liver. In both liver phospholipids and triglycerides, the two sole long-chain monodesaturated $\omega 9$ fatty acids present in sizeable amounts were $\mathrm{C} 18: 1 \omega 9$ and $\mathrm{C} 20: 1 \omega 9$. The relative weight content of $\mathrm{C} 20: 1 \omega 9$ (per thousand) in liver phospholipids was $70 \%$ higher $(\mathrm{p}<0.01)$ in fed female STZ rats than that found, when detected, in fed female control animals (Table I). In the female GK rats, however, such a C20:1w9 relative content failed to differ significantly $(\mathrm{p}>0.6)$ from that found in control animals, the measurements made in GK rats averaging $103.4 \pm 4.2 \%(n=4)$ of the mean corresponding values found in control animals of the same gender (female) and nutritional status (fed or fasted), i.e. $100.0 \pm 4.8 \%(n=6)$. In both control and GK rats, the C20:1 $1 \omega 9$ relative content of liver phospholipids was much lower $(\mathrm{p}<0.001)$ in fed than in starved rats, the values recorded in the fed animals averaging $60.6 \pm 4.1 \%(n=6)$ of the mean corresponding measurements made in starved rats of the same gender (male or female) and 


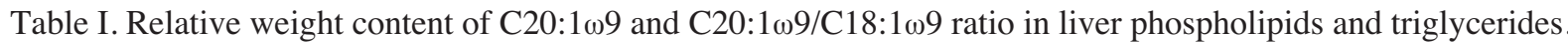

\begin{tabular}{|c|c|c|c|c|}
\hline \multirow[t]{2}{*}{ Rats } & \multicolumn{2}{|c|}{ Phospholipids } & \multicolumn{2}{|c|}{ Triglycerides } \\
\hline & $\mathrm{C} 20: 1 \omega 9(\%)$ & $\mathrm{C} 20: 1 \omega 9 / \mathrm{C} 18: 1 \omega 9(\%)$ & $\mathrm{C} 20: 1 \omega 9(\%)$ & $\mathrm{C} 20: 1 \omega 9 / \mathrm{C} 18: 1 \omega 9(\%)$ \\
\hline Fed female control & $0.58 \pm 0.08$ & $20.1 \pm 1.0(2)$ & $2.44 \pm 0.50(3)$ & $8.1 \pm 1.3(3)$ \\
\hline Fasted female control & $0.98 \pm 0.05(4)$ & $55.3 \pm 4.2(4)$ & $2.11 \pm 0.24$ & $15.4 \pm 2.6(4)$ \\
\hline Fed female STZ & $0.98 \pm 0.05(5)$ & $45.9 \pm 1.8(5)$ & $1.93 \pm 0.21(6)$ & $13.2 \pm 2.0(6)$ \\
\hline Fed female GK & $0.57 \pm 0.05$ & $32.7 \pm 5.4(2)$ & $2.36 \pm 0.67(2)$ & $9.3 \pm 2.3(2)$ \\
\hline Fasted female GK & $1.05 \pm 0.01(2)$ & $61.1 \pm 1.8(2)$ & $1.57(1)$ & $7.8(1)$ \\
\hline Fed male GK & $1.06 \pm 0.12(2)$ & $37.8 \pm 1.2(2)$ & $1.42 \pm 0.01$ & $8.0 \pm 0.1(2)$ \\
\hline Fasted male GK & $1.55 \pm 0.02(2)$ & $57.8 \pm 3.2(2)$ & $1.79 \pm 0.04(2)$ & $13.5 \pm 0.0(2)$ \\
\hline
\end{tabular}

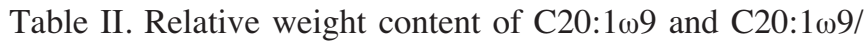
C18:1 109 ratio in brain phospholipids.

\begin{tabular}{|c|c|c|}
\hline Rats & $\mathrm{C} 20: 1 \omega 9(\%)$ & $\begin{array}{c}\mathrm{C} 20: 1 \omega 9 / \\
\mathrm{C} 18: 1 \omega 9(\%)\end{array}$ \\
\hline Fed female control & $2.49 \pm 0.43(4)$ & $18.3 \pm 2.5$ \\
\hline Fasted female control & $7.65 \pm 0.41(4)$ & $52.0 \pm 1.0$ \\
\hline Fed female STZ & $8.01 \pm 0.57(6)$ & $56.1 \pm 3.5$ \\
\hline Fed female GK & $9.61 \pm 6.20(2)$ & $51.5 \pm 27.7(2)$ \\
\hline Fasted female GK & $3.64 \pm 0.10(2)$ & $24.1 \pm 0.1$ \\
\hline Fed male GK & $6.99 \pm 4.58(2)$ & $45.8 \pm 27.3(2)$ \\
\hline Fasted male GK & $19.54 \pm 0.07(2)$ & $104.1 \pm 3.8$ \\
\hline
\end{tabular}

same strain (control or GK), i.e. $100.0 \pm 2.4 \%(n=8)$. Last, in the GK rats, the data collected in male rats averaged $166.4 \pm 13.8 \%(n=4 ; p<0.005)$ of the mean corresponding values found in female rats in the same nutritional status (fed or fasted), i.e. $100.0 \pm 3.6 \%(n=4)$.

The $\mathrm{C} 20: 1 \omega 9 / \mathrm{C} 18: 1 \omega 9$ ratio in liver phospholipids provided information somewhat different from that just mentioned and concerning the relative contribution of C20:1 $\omega 9$. First, in the GK rats, no gender difference was anymore observed, the $\mathrm{C} 20: 1 \omega 9 / \mathrm{C} 18: 1 \omega 9$ ratio averaging in the male rats $105.1 \pm 6.6 \%$ $(\mathrm{n}=4 ; \mathrm{p}>0.5)$ of the corresponding means values recorded in female rats $(100.0 \pm 5.6 \%$; $n=4)$ examined in the same nutritional status (fed or fasted). Second, whilst the measurements made in fed rats remained significantly lower than those recorded in fasted rats, whether in control animals $(36.4 \pm 1.8 \%$; $n=2$ versus $100.0 \pm 7.6 \% ; \mathrm{n}=4 ; \mathrm{p}<0.006)$ or $\mathrm{GK}$ rats $(59.4 \pm 5.0 \%$, $\mathrm{n}=4$ versus $100.0 \pm 2.3 \%, \mathrm{n}=4 ; \mathrm{p}<0.001)$, such a difference was, in relative terms, less pronounced $(\mathrm{p}<0.05)$ in GK rats than in control animals. Last, whilst the normalized values recorded in fed GK rats $(174.9 \pm 13.3 \%, n=4)$ were significantly higher than those found in fed control animals $(100.0 \pm 5.0 \% ; n=2)$, such was not the case $(\mathrm{p}>0.4)$ when comparing starved GK rats $(107.4 \pm 3.2 \% ; n=4)$ to starved control animals $(100.0 \pm 7.6 \% ; n=4)$. In fed STZ rats, like in the fed GK rats, the $\mathrm{C} 20: 1 \omega 9 / \mathrm{C} 18: 1 \omega 9$ ratio remained higher $(0.046 \pm 0.002$; $\mathrm{n}=5)$ than in fed control animals $(0.020 \pm 0.001 ; \mathrm{n}=2)$.

The weight per thousand of C20:1 $\omega 9$ in liver triglycerides failed to differ significantly in female control animals
$(2.25 \pm 0.24 \%$; $n=7)$, female STZ rats $(1.93 \pm 0.21 \%$; $n=6)$ and female GK rats $(2.09 \pm 0.47 \%$; $n=3)$. No significant difference was found between fed and fasted rats, and between male and female GK rats.

Even the C20:1 $\omega 9 / C 18: 1 \omega 9$ ratio failed to differ significantly in fed versus fasted rats $(73.8 \pm 13.9 \%$ versus $100.0 \pm 9.2 \%$, d.f. $=12, \mathrm{p}>0.1)$, in male versus female GK rats (129.9 $\pm 25.3 \%$ versus $100.0 \pm 14.3 \%$, d.f. $=5, \mathrm{p}>0.3)$, in female fed STZ rats versus female fed control animals $(162.8 \pm 24.5 \%$ versus $100.0 \pm 15.9 \%$; d.f. $=7, \mathrm{p}>0.1$ ), and in GK rats versus control animals examined in the same nutritional status $(93.5 \pm 10.3 \%$ versus $100.0 \pm 11.0 \%$, d.f. $=12, \mathrm{p}>0.6)$.

The C20:1 $\omega 9 / \mathrm{C} 18: 1 \omega 9$ ratio was always much lower in liver triglycerides than in liver phospholipids, averaging in the former case $28.2 \pm 2.3 \%(n=20 ; p<0.001)$ of the mean corresponding values found in the liver phospholipids of the same group of rats $(100.0 \pm 2.2 \% ; \mathrm{n}=19)$.

Brain. A sizeable amount of C22:1 19 in brain phospholipids was only detected in one fasted female control rat $(1.84 \%$ ) and two fasted male GK rats $(3.33 \pm 0.23 \%$ o).

Like in liver, the C20:1 $\omega 9$ content of brain phospholipids was significantly higher $(\mathrm{p}<0.001)$ in female starved control or female fed STZ rats than in female fed control rats (Table II). It was also significantly higher $(\mathrm{p}<0.001)$ in male than female GK rats examined after overnight starvation. However, in both male and female fed GK rats, the C20:1 199 content of brain phospholipids yielded two vastly different individual values. The C20:1 $19 / \mathrm{C} 18: 1 \omega 9$ paired ratio in brain phospholipids yielded the same information as that just mentioned and concerning the relative contribution of $\mathrm{C} 20: 1 \omega 9$ in brain phospholipids (Table II).

As expected from these findings, there was a significant positive correlation $(\mathrm{r}=0.4241, \mathrm{n}=22, \mathrm{p}<0.05)$ between the individual values for the $\mathrm{C} 20: 1 \omega 9 / \mathrm{C} 18: 1 \omega 9$ ratio in liver and brain. Such a correlation was most obvious, however, in the Wistar female rats, at the exclusion of all GK rats, yielding a correlation coefficient of $0.7558(n=14, p<0.001)$.

Except in 2 out of 22 rats, no C20:1w9 was detected in brain triglycerides.

Spleen. The C20:1 $1 \omega 9$ relative weight content of spleen phospholipids failed to differ significantly in fed female control rats and either fasted female control rats or fed female STZ rats. 


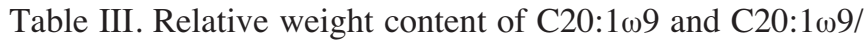
C18:1 109 ratio in spleen phospholipids.

\begin{tabular}{lcc}
\hline Rats & $\mathrm{C} 20: 1 \omega 9(\%)$ & $\begin{array}{c}\mathrm{C} 20: 1 \omega 9 / \\
\mathrm{C} 18: 1 \omega 9(\%)\end{array}$ \\
\hline Fed female control & $1.63 \pm 0.08(4)$ & $24.1 \pm 1.2(4)$ \\
Fasted female control & $1.54 \pm 0.05(4)$ & $26.6 \pm 1.0(4)$ \\
Fed female STZ & $1.73 \pm 0.09(6)$ & $30.0 \pm 1.3(6)$ \\
Fed female GK & $1.70 \pm 0.10(2)$ & $29.4 \pm 2.3(2)$ \\
Fasted female GK & $1.60 \pm 0.07(2)$ & $25.0 \pm 0.4(2)$ \\
Fed male GK & $1.80 \pm 0.01(2)$ & $33.5 \pm 0.6(2)$ \\
Fasted male GK & $1.66 \pm 0.02(2)$ & $29.5 \pm 0.2(2)$ \\
\hline
\end{tabular}

It also failed to differ significantly between female control versus GK rats and between female versus male GK rats (Table III).

The $\mathrm{C} 20: 1 \omega 9 / \mathrm{C} 18: 1 \omega 9$ ratio of spleen phospholipids also displayed much less pronounced variations than those observed in liver or brain. Nevertheless, in the spleen phospholipids, such a ratio was significantly higher in fed female STZ rats than in fed female control rats $(p<0.02)$, in male than in female GK rats examined in the same nutritional state $(116.1 \pm 1.5$ versus $100.0 \pm 3.2 \%, \mathrm{n}=4$ in both cases, $\mathrm{p}<0.005$ ), and in fed rather than fasted GK rats of the same gender $(115.6 \pm 4.0$ versus $100.0 \pm 0.7 \%, \mathrm{n}=4$ in both cases, $\mathrm{p}<0.01)$. Unexpectedly, the value found in the fed GK rats, expressed relative to that found in fasted GK rats of the same gender $(115.6 \pm 4.0 \%$, $\mathrm{n}=4)$, was significantly different $(\mathrm{p}<0.01)$ from that recorded in fed control rats, also expressed relative to that found in fasted control rats of the same gender $(90.5 \pm 4.5 \% ; n=4)$.

A sizeable amount of $\mathrm{C} 20: 1 \omega 9$ in spleen triglycerides was only detected in 6 out of 22 animals, with a mean value of $0.22 \pm 0.02 \%(\mathrm{n}=6)$.

Adipose tissue. In the parametrial adipose tissue, the relative weight contribution of $\mathrm{C} 20: 1 \omega 9$ to the total fatty acid content of lipids failed to differ significantly in fed versus fasted rats. It averaged $2.14 \pm 0.33 \%$ o $(\mathrm{n}=4)$ in fed female control rats, $2.41 \pm 0.29 \%$ o $(n=4)$ in overnight starved female control rats, and $2.16 \pm 0.10 \%(\mathrm{n}=6)$ in fed female STZ. It was somewhat lower $(\mathrm{p}<0.005)$, however, in female GK rats $(1.41 \pm 0.07 \%$; $\mathrm{n}=4$ ), as well as in the epididymal adipose tissue of male GK rats $(1.54 \pm 0.11 \%$; $\mathrm{n}=4)$.

Likewise, in the lipids of adipose tissue, the C20:1 $109 /$ C18:1 $\omega 9$ ratio failed to differ significantly in fed female control rats, fasted female control rats and fed female STZ rats (Table IV), yielding an overall mean value of $9.26 \pm 0.54$ $\% o(\mathrm{n}=14)$. It was lower $(\mathrm{p}<0.005)$ in the female GK rats $(5.07 \pm 0.30 \% ; n=4)$, in which case the $\mathrm{C} 20: 1 \omega 9 / \mathrm{C} 18: 1 \omega 9$ ratio found in parametrial fat was also lower $(p<0.05)$ than that recorded in the epididymal adipose tissue of male GK rats $(6.51 \pm 0.49 ; n=4)$. No significant effect of the nutritional status (fed versus overnight fasted rats) was detected in any animal group. The results recorded in fed rats indeed averaged $100.0 \pm 7.6 \%(n=8)$ of the mean corresponding values found in starved animals of the same strain and gender $(100.0 \pm 7.2 \%$; $n=8)$.

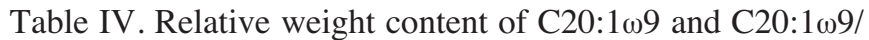
C18:1 $\omega 9$ ratio in the lipids of parametrial or epididymal adipose tissue.

\begin{tabular}{lcc}
\hline Rats & $\mathrm{C} 20: 1 \omega 9(\%)$ & $\begin{array}{c}\mathrm{C} 20: 1 \omega 9 / \\
\mathrm{C} 18: 1 \omega 9(\%)\end{array}$ \\
\hline Fed female control & $2.14 \pm 0.33(4)$ & $9.8 \pm 1.6(4)$ \\
Fasted female control & $2.41 \pm 0.29(4)$ & $9.6 \pm 1.2(4)$ \\
Fed female STZ & $2.16 \pm 0.10(6)$ & $8.6 \pm 0.3(6)$ \\
Fed female GK & $1.36 \pm 0.05(2)$ & $5.0 \pm 0.2(2)$ \\
Fasted female GK & $1.47 \pm 0.15(2)$ & $5.2 \pm 0.7(2)$ \\
Fed male GK & $1.46 \pm 0.01(2)$ & $6.5 \pm 0.2(2)$ \\
Fasted male GK & $1.61 \pm 0.23(2)$ & $6.5 \pm 1.2(2)$ \\
\hline
\end{tabular}

No significant correlation between liver triglycerides and adipose tissue lipids was observed as far as the individual values for either the C20:1 $\omega 9$ relative weight content $(\mathrm{r}=0.1260, \mathrm{n}=22, \mathrm{p}>0.1)$ or $\mathrm{C} 20: 1 \omega 9 / \mathrm{C} 18: 1 \omega 9$ ratio $(\mathrm{r}=0.2573$, $\mathrm{n}=22, \mathrm{p}>0.1)$ are concerned.

Plasma. Several features of the liver phospholipid C20:1 19 relative content were also observed in plasma phospholipids. For instance, when present in detectable amount, the values for such a content recorded in fed rats were $30.6 \pm 10.6 \%$ lower (d.f. $=7, p<0.025)$ than those found in starved rats of the same strain and gender. Likewise, the $\mathrm{C} 20: 1 \omega 9$ relative content of plasma phospholipids was $50.1 \pm 19.9 \%$ higher (d.f. $=4 ; \mathrm{p}<0.07$ ) in male than female GK rats examined in the same nutritional state. As a matter of fact and as shown in Fig. 1, a highly significant positive correlation was found between the individual values for the $\mathrm{C} 20: 1 \omega 9$ relative content of phospholipids in liver and plasma $(r=0.6653, n=22$, $\mathrm{p}<0.001)$.

Likewise, the modulation of the $\mathrm{C} 20: 1 \omega 9 / \mathrm{C} 18: 1 \omega 9$ ratio in plasma phospholipids was similar to that identified in liver phospholipids (Table V). For instance, the values found in fed rats were $50.1 \pm 4.5 \%$ lower (d.f. $=7, p<0.001$ ) than those recorded in fasted animals of the same strain and gender. Also in fair agreement with the liver data, no significant gender difference $(\mathrm{p}>0.4)$ was anymore observed in the GK rats for the $\mathrm{C} 20: 1 \omega 9 / \mathrm{C} 18: 1 \omega 9$ plasma phospholipid ratio. As illustrated in Fig. 1, there was indeed a highly significant positive correlation between the individual values for the C20:1 $\omega 9 / \mathrm{C} 18: 1 \omega 9$ ratio in the liver and plasma phospholipids $(\mathrm{r}=0.6272, \mathrm{n}=22, \mathrm{p}<0.003)$. The absolute value for such a ratio was $60.0 \pm 8.8 \%$ higher $(\mathrm{d} . \mathrm{f} .=29 ; \mathrm{p}<0.001)$, however, in the plasma phospholipids than in the liver phospholipids of the same type(s) of rats.

In sharp contrast, no significant correlation between liver and plasma individual data was observed when considering either the relative weight content of $\mathrm{C} 20: 1 \omega 9$ in triglycerides $(\mathrm{r}=0.1229, \mathrm{n}=22, \mathrm{p}>0.1)$ or the triglyceride $\mathrm{C} 20: 1 \omega 9 /$ $\mathrm{C} 18: 1 \omega 9$ ratio $(\mathrm{r}=0.3804, \mathrm{n}=22, \mathrm{p}>0.05)$. The latter ratio was again about twice higher $(198.1 \%$; d.f. $=42 ; \mathrm{p}<0.05)$ in the plasma triglycerides than in the liver triglycerides of the same type(s) of rats (Fig. 2). 

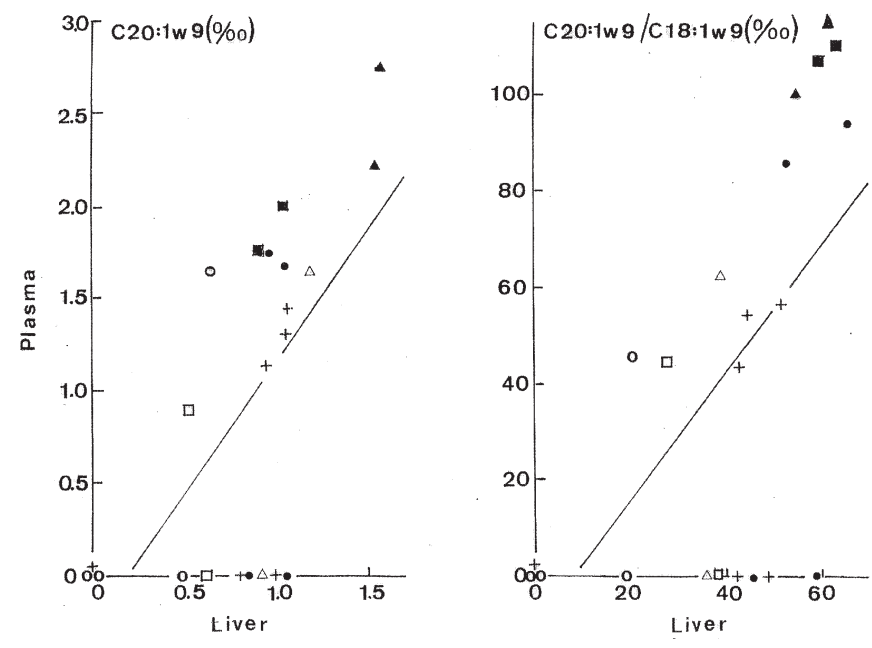

Figure 1. Correlation between the plasma and liver values for the C20:1 09 relative weight content (left) and $\mathrm{C} 20: 1 \omega 9 / \mathrm{C} 18: 1 \omega 9$ ratio (right) of phospholipids in fed (open symbols) or overnight fasted (close symbols) female control rats (circles), female GK rats (squares) and male GK rats (triangles), as well as fed female STZ rats (crosses). The oblique lines correspond to the regression lines.

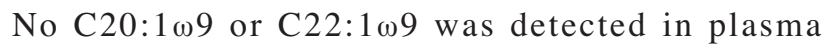
unesterified fatty acids in any of the 22 examined in this study.

\section{Discussion}

The methodological validity of the analytical procedure used in the present work was recently documented in an independent study conducted, inter alia, in four groups of 4-12 fed female control rats (8).

The present study deals mainly with three sets of observations.

First, it reveals that, in the liver, brain and plasma phospholipids, the $\mathrm{C} 20: 1 \omega 9$ relative content is lower in fed rats than in overnight fasted rats. In control and GK rats, the fed/fasted ratio averaged $60.6 \pm 4.5 \%$ (d.f. $=12$ ) in the liver, $42.3 \pm 10.1 \%$ (d.f.=13) in the brain after exclusion of one abnormally high individual value found in a female fed GK rat, and $69.8 \pm 10.6 \%$ (d.f.=7) in the plasma ( $<<0.025$ or less in all cases). A comparable nutritional difference was not observed in spleen
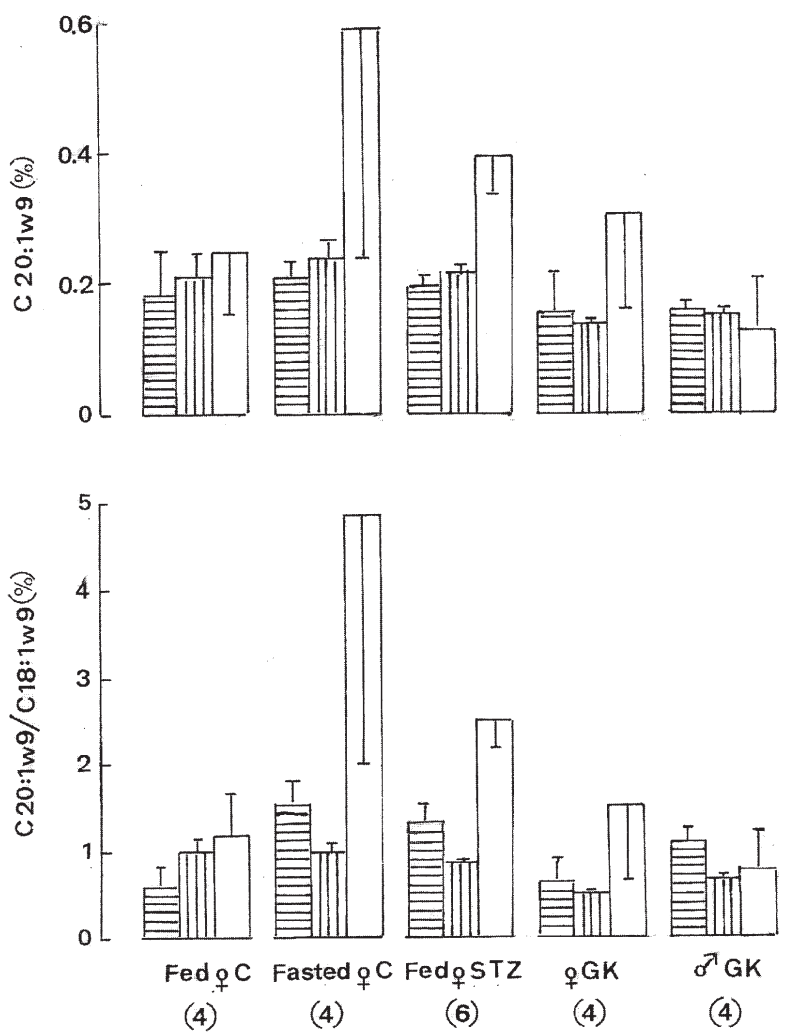


C18:1 $\omega 9$ ratio (lower panel) in liver triglycerides (horizontally hatched columns), adipose tissue lipids (vertically hatched columns) and plasma triglycerides (open columns) of fed or fasted female control (C) rats, fed female STZ rats, and both fed and fasted female or male GK rats. Mean values $( \pm$ SEM) refer to the number of individual observations indicated in parentheses at the bottom of the figure.

phospholipids. The fed/fasted ratio recorded in liver phospholipids also differed $(\mathrm{p}<0.01)$ from that found in liver triglycerides, i.e. $115.2 \pm 18.0 \%$. Likewise, in the adipose tissue lipids, the C20:1 $\omega 9$ relative content was not significantly different in fed and overnight fasted rats, with a fed/fasted ratio of $90.2 \pm 9.1 \%$ (d.f. $=14 ; \mathrm{p}=0.3$ ).

Second, the data collected in GK rats indicate that a gender difference was not uncommon. For instance, in liver, brain and plasma, the mean $\mathrm{C} 20: 1 \omega 9$ relative content of phospho-

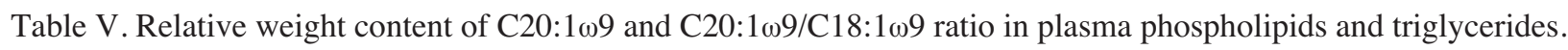

\begin{tabular}{|c|c|c|c|c|}
\hline \multirow[t]{2}{*}{ Rats } & \multicolumn{2}{|c|}{ Phospholipids } & \multicolumn{2}{|c|}{ Triglycerides } \\
\hline & C20:1w9 (\%o) & 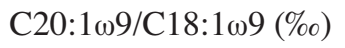 & $\mathrm{C} 20: 1 \omega 9(\%)$ & $\mathrm{C} 20: 1 \omega 9 / \mathrm{C} 18: 1 \omega 9(\%)$ \\
\hline Fed female control & $1.64(1)$ & $45.8(1)$ & $3.32 \pm 0.64(3)$ & $15.6 \pm 4.3(3)$ \\
\hline Fasted female control & $1.71 \pm 0.04(2)$ & $90.0 \pm 3.9(2)$ & $11.81 \pm 1.86(2)$ & $97.0 \pm 12.0(2)$ \\
\hline Fed female STZ & $1.29 \pm 0.08$ & $51.8 \pm 4.0(3)$ & $3.97 \pm 0.59(6)$ & $24.9 \pm 3.2(6)$ \\
\hline Fed female GK & $0.89(1)$ & $44.2(1)$ & $2.84 \pm 1.34(2)$ & $12.1 \pm 6.0(2)$ \\
\hline Fasted female GK & $1.88 \pm 0.12(2)$ & $108.5 \pm 1.7(2)$ & $6.62(1)$ & $39.4(1)$ \\
\hline Fed male GK & $1.64(1)$ & $62.6(1)$ & $2.61 \pm 0.37(2)$ & $15.3 \pm 1.9(2)$ \\
\hline Fasted male GK & $2.48 \pm 0.26(2)$ & $107.6 \pm 7.2(2)$ & N.D. & N.D. \\
\hline
\end{tabular}

N.D., not detected in any rat. 
lipids was lower in female than in male rats examined in the same nutritional state, with an overall female/male ratio of $53.5 \pm 11.4 \%$ (d.f. $=19 ; \mathrm{p}<0.001$ ), always after exclusion of the abnormally high value found in the brain of a single female fed GK rat. Once again, such a gender difference was not observed in spleen phospholipids. It also failed to be observed in either liver triglycerides or adipose tissue lipids, with an overall female/male ratio of $112.7 \pm 16.4 \%$ (d.f. $=13 ; \mathrm{p}>0.4$ ).

Last, the present study reveals diabetes-related changes in the variables under consideration. Thus, in liver and brain, the phospholipid $\mathrm{C} 20: 1 \omega 9 / \mathrm{C} 18: 1 \omega 9$ ratio averaged, in fed

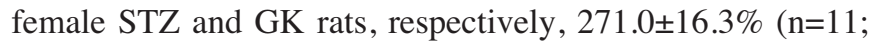
$\mathrm{p}<0.001)$ and $222.1 \pm 71.5 \%(\mathrm{n}=4 ; \mathrm{p}<0.07)$ of the mean corresponding values found in fed female control rats $(100.0 \pm 8.7 \%$; $n=6)$. The just mentioned percentages failed to differ significantly $(\mathrm{p}>0.3)$ in STZ and GK rats, yielding an overall mean value $(258.0 \pm 21.6 \% ; n=15)$ much higher $(\mathrm{p}<0.001)$ than that recorded in control rats of the same gender examined in the same nutritional state. Once again, a comparable situation was not observed in spleen phospholipids, in which case the $\mathrm{C} 20: 1 \omega 9 / \mathrm{C} 18: 1 \omega 9$ ratio found in fed female STZ and GK rats represented no more than $78.6 \pm 6.4 \%$ $(\mathrm{n}=8, \mathrm{p}>0.15)$ of that recorded in fed female control rats $(100.0 \pm 16.3 \%$; $n=4)$. Likewise the diabetic/control value for the $\mathrm{C} 20: 1 \omega 9 / \mathrm{C} 18: 1 \omega 9$ ratio failed to differ significantly from unity, whether in liver triglycerides $(83.5 \pm 18.5 \%$; d.f. $=9$; $\mathrm{p}>0.3)$, plasma triglycerides $(111.1 \pm 29.1 \%$; d.f. $=9$; $\mathrm{p}>0.7)$ or parametrial adipose tissue lipids $(91.6 \pm 14.5 \%$; d.f. $=10$; $\mathrm{p}>0.5)$. At the most, there was a trend $(\mathrm{p}<0.025)$ for a higher triglyceride $\mathrm{C} 20: 1 \omega 9 / \mathrm{C} 18: 1 \omega 9$ ratio in the liver and plasma of STZ rats $(161.3 \pm 15.3 \% ; \mathrm{n}=12)$, as compared to control animals $(100.0 \pm 14.3 \% ; \mathrm{n}=6)$.

The differences invariably observed between liver and brain phospholipids versus spleen phospholipids, on one hand, and versus liver triglycerides or adipose tissue lipids, on the other hand, are reminiscent of prior observations indicating organ-specific changes in the fatty acid pattern of phospholipids, as distinct from triglycerides $(6,9)$.

The present study also allows to extend to C20:1w9 the proposal that the liver phospholipid relative content in a given fatty acid may be judged from measurements conducted in plasma phospholipids (10), at least when comparing the mean values recorded in distinct groups of animals.

The question remains whether the high C20:1 $19 / \mathrm{C} 18: 1 \omega 9$ ratio found in the liver phospholipids of diabetic animals reflects an accelerated generation of C20:1 109 from $\mathrm{C} 18: 1 \omega 9$, as catalyzed by elongase, or an impaired conversion of $\mathrm{C} 20: 1 \omega 9$ to further long-chain monodesaturated $\omega 9$ fatty acids. In this respect, it should be underlined that the increased C20:1 $\omega 9 / \mathrm{C} 18: 1 \omega 9$ ratio found in the liver phospholipids of female diabetic rats represents a mirror image of that recently documented in male diabetic rats (8). Such contrasting results could conceivably account for the observation that, in either 12 insulin-dependent or 8 non-insulin-dependent diabetic

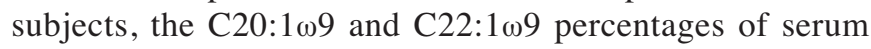
phosphaditylcholine and sphingomyelin failed to differ significantly from those found in 29 control subjects (11). Indeed, the results of the latter study refer to both men and women, with a mean male/female sex ratio of $1.36 \pm 0.26$ $(n=3 ; p>0.2$ versus unity).
In conclusion, when taken in consideration together with the findings recently collected in male STZ and GK rats, the present results suggest a gender difference in the diabetesinduced changes for the pattern of long-chain monodesaturated $\omega 9$ fatty acids in liver phospholipids. Such a difference coincides with the knowledge (12) that the adjusted hazard ratio for coronary heart disease, is much higher in women (1.97) than in men (1.17).

\section{Acknowledgements}

This study was supported in part by a grant (3.4574.07) from the Belgian Foundation for Scientific Medical Research. We are grateful to A. Chwalik and A. Dufour for technical assistance, and to C. Demesmaeker for secretarial help.

\section{References}

1. Oda E, Harada K, Kimura J, Aizawa Y, Thanikachalam PV and Watanabe K: Relationships between serum unsaturated fatty acids and coronary risk factors: negative relations between nervonic acid and obesity-related risk factors. Int Heart $\mathrm{J} 46$ : 975-985, 2005.

2. Malaisse WJ, Portois L, Zhang Y, Oguzhan B, Louchami K, Jijakli H, Courtois P, Sener A and Carpentier YA: Fatty acid content and pattern of epididymal and parametrial adipose tissue lipids in streptozotocin (type 1) and Goto-Kakizaki (type 2) diabetic rats. Int J Mol Med 18: 1231-1234, 2006.

3. Malaisse WJ, Zhang Y, Oguzhan B, Louchami K, Jijakli H, Courtois P, Sener A, Portois L and Carpentier YA: Anomalies of liver and plasma triglyceride and phospholipid fatty acid pattern in streptozotocin (type 1) and Goto-Kakizaki (type 2) diabetic rats. Res Rev Biosci 78-86, 2007.

4. Malaisse WJ, Zhang Y, Oguzhan B, Louchami K, Jijakli H, Courtois P, Sener A, Portois L and Carpentier YA: Perturbation of desaturase- and elongase-catalyzed equilibrium between fatty acids in liver and plasma triglycerides and phospholipids in streptozotocin (type 1) and Goto-Kakizaki (type 2) diabetic rats. Res Rev Biosci 87-98, 2007.

5. Portois L, Sener A, Carpentier YA and Malaisse WJ: Fatty acid content and pattern of spleen phospholipids and triglycerides in normal and either Type- 1 or Type- 2 diabetic rats. Int J Mol Med 19: 523-528, 2007.

6. Malaisse WJ, Zhang Y, Louchami K, Sener A, Portois L and Carpentier YA: Brain phospholipid and triglyceride fatty acid content and pattern in Type 1 and Type 2 diabetic rats. Neurosci Lett 409: 75-79, 2006.

7. Richelle M, Carpentier YA and Deckelbaum RJ: Long- and medium-chain triacylglycerols in neutral lipid-exchange processes with human plasma low-density lipoproteins. Biochemistry 33: 4872-4878, 1984

8. Malaisse WJ, Zhang Y, Portois L, Sener A and Carpentier YA: Liver phospholipid and triglyceride content of 11-eicosenoic and 13-docosenoic acids: gender difference and perturbation in type 1 and type 2 diabetic rats. Metab Func Res Diabetes (In press).

9. Carpentier YA, Portois L, Sener A and Malaisse WJ: Age-related perturbations of plasma lipid fatty acid content and pattern in $\omega 3$-depleted rats examined before and shortly after the intravenous injection of two distinct lipid emulsions. Trends Cell Mol Biol (In press).

10. Carpentier YA, Peltier S, Portois L, Sener A and Malaisse WJ: Rapid lipid enrichment in $\omega 3$ fatty acids: Plasma data. Int J Mol Med 21: 355-365, 2008.

11. Seigneur M, Freyburger G, Gin H, Claverie M, Lardeau D, Lacape G, Le Moigne F, Crockett R and Boisseau MR: Serum fatty acid profiles in type I and II diabetes: metabolic alterations of fatty acids of the main serum lipids. Diabetes Res Clin Pract 23: 169-177, 1994.

12. Whitelay L, Padmanabhan S, Hole D and Isles C: Should diabetes be considered a coronary heart disease risk equivalent? Diabetes Care 28: 1588-1593, 2005. 